\title{
T-Stability of General One-Step Methods for Abstract Initial-Value Problems
}

\author{
István Faragó $^{1,2, *}$ and Imre Fekete ${ }^{1,2, *}$ \\ ${ }^{I}$ Department of Applied Analysis and Computational Mathematics, Eötvös Loránd, University, Pázmány P. s. 1/C, 1117, \\ Budapest, Hungary; ${ }^{2}$ MTA-ELTE Numerical Analysis and Large Networks Research Group, Pázmány P. s., 1/C, 1117, \\ Budapest, Hungary
}

\begin{abstract}
In this paper we investigate the T-stability of one-step methods for initial-value problems. The main result is that we extend the classical result (the well-known Euler method) for variable step size explicit and implicit one-step methods. In addition, we give further properties for the theory of T-stability of nonlinear equations in an abstract (Banach space) setting.
\end{abstract}

Keywords: Finite difference method, stability, convergence, Euler method, operator equation.

\section{INTRODUCTION}

Many phenomena in nature can be described by mathematical models. Typically, the solution of such models cannot be given in a closed (analytical) form, therefore we construct numerical models in order to approximate the exact solution. The investigation of the numerical solution of nonlinear operator equations in an abstract (Banach space) setting has been done in several works, e.g., in Keller [4], Stetter [7] and many others.

Roughly speaking, the consistency is the characterization of the local (truncation) error of the method, which is the error committed by one step of the method. (It is the difference between the result given by the method, assuming that no error was made in earlier steps, and the exact solution.) On the other hand, the stability guarantees that the numerical method produces a bounded solution whenever the solution of the exact differential equation is bounded, in other words, the local truncation errors are damped out. Convergence means that the numerical solution approximates the solution of the original problem, i.e., a numerical method is said to be convergent if the numerical solution approaches the exact solution as the discretization parameter (usually the step size of the mesh) goes to zero.

The basic problem is the following. The definition of convergence assumes the knowledge of the solution of the original (continuous) problem, therefore it cannot be verified directly. Our aim is its replacement with simpler conditions. The main result is that the convergence can be guaranteed by two, directly verifiable conditions, which are consistency and stability.

In this context the suitable choice of the stability plays a crucial role: it should be chosen to guarantee the

*Address correspondence to these authors at the Department of Applied Analysis and Computational Mathematics, Eötvös Loránd, University, Pázmány P. s. 1/C, 1117, Budapest, Hungary; Tel: 36-1-372-2500/8436;

Fax:36-1-381-2158; Emails: faragois@cs.elte.hu and feipaat@cs.elte.hu convergence (together with the consistency), moreover, that the condition can be verified in the applications. The relationship between stability and convergence for linear problems, hinted by Courant, Friedrichs and Lewy in the 1920's ([2]), identified more clearly by von Neumann [1] in the 1940's and brought into organized form by Lax and Richtmyer in the 1950's as the Lax (alternatively, LaxRichtmyer-Kantorovich) equivalence theorem (Lax, Richtmyer [6]), which states that for consistent numerical approximations, stability and convergence are equivalent. However, the stability notion for nonlinear problems are less investigated.

Unfortunately, von Neumann's stability is necessary and sufficient for stability in the sense of the Lax equivalence theorem only in certain cases: the models must be linear, the considered partial differential equation must be constantcoefficient with periodic boundary conditions, etc. However, due to its relative simplicity it is often used in place of a more detailed stability analysis as a good guess at the restrictions (if any) on the step sizes used in the scheme.

Stability, in general, can be difficult to investigate, especially when the equation under consideration is nonlinear.

The various nonlinear stability notions (Keller [4], Stetter [7], Trenogin [8]) are useful for theoretical results, but it is not straightforward to verify them. As for the application of these stability notions, they are applied to the well-known initial-value problem where the right-hand side function $f$ is globally Lipschitzian. In [3] we analyzed the explicit Euler method (EE method) on uniform grid and also its S-stability (and thus K-stability, too) for this problem. It has been shown that this method is T-stable, too (see [8]). In this work we have also considered the nonlinear abstract setting, and we showed that for different nonlinear stability notions the conditions of consistency and stability together are a sufficient, but not a necessary condition for the convergence. For the applied discretization we have considered the same model: explicit Euler method on uniform mesh. 
In this paper we generalize our results. We will show that for the above described initial-value problem a much wider class of numerical methods is T-stable. Namely, we prove that the explicit and implicit one-step methods, including both the explicit and implicit Runge-Kutta methods (ERK, IRK methods) are T-stable on both uniform and non-uniform grids.

The paper is organized as follows. In Section 2 we give the mathematical formulation and a general description of the mathematical and numerical models. Moreover, we define the basic numerical notions (convergence, consistency). In Section 3 we introduce the notion of Tstability based on the work of Trenogin, we formulate the classical results and add further properties. In Section 4 we verify the T-stability for initial-value problems for the above mentioned cases. In Section 5 we make remarks and draw conclusions.

\section{MATHEMATICAL BACKGROUND}

We consider a given problem of the form

$$
F(u)=0 \text {, }
$$

where $F: D \rightarrow Y$ is a (nonlinear) operator, $\mathrm{D} \subset \mathrm{X}, \mathrm{X}$ and $Y$ are normed spaces. In the theory of numerical analysis it is usually assumed that there exists a unique solution, which will be denoted by $\bar{u}$.

\section{Definition 1}

Problem (1) can be given as a triplet $\mathrm{P}=(\mathrm{X}, \mathrm{Y}, F)$. We will refer to it as problem $\mathrm{P}$.

Definition $2 \mathrm{We}$ say that the sequence $\mathrm{N}=\left(\mathrm{X}_{n}, \mathrm{Y}_{n}, F_{n}\right)_{n \in \mathrm{N}}$ is a numerical method if it generates a sequence of problems

$F_{n}\left(u_{n}\right)=0, \quad n=1,2, \ldots$,

where

- $\mathrm{X}_{n}, \mathrm{Y}_{n}$ are normed spaces,

- $\mathrm{D}_{n} \subset \mathrm{X}_{n}$ and $F_{n}: \mathrm{D}_{n} \rightarrow \mathrm{Y}_{n}$.

If there exists a unique solution of the (approximating) problems (2), it will be denoted by $\bar{u}_{n}$.

Definition 3 We say that the sequence $\mathrm{D}=\left(\varphi_{n}, \psi_{n}, \Phi_{n}\right)_{n \in \mathrm{N}}$ is a discretization if

- the $\varphi_{n}$-s (respectively $\psi_{n}$-s) are restriction operators from $X$ into $X_{n}$ (respectively from $Y$ into $Y_{n}$ ), where $\mathrm{X}, \mathrm{X}_{n}, \mathrm{Y}, \mathrm{Y}_{n}$ are normed spaces,

- $\Phi_{n}:\{F: \mathrm{D} \rightarrow \mathrm{Y} \mid \mathrm{D} \subset \mathrm{X}\} \rightarrow\left\{F_{n}: \mathrm{D}_{n} \rightarrow \mathrm{Y}_{n} \mid \mathrm{D}_{n} \subset \mathrm{X}_{n}\right\}$.

In the sense of this definition we can imagine the general scheme in the following way, as shown in (Fig. 1):

Assumption 4 The discretization $D$ possesses the property $\psi_{n}(0)=0$.

Assumption 5 The discretization D generates a numerical method $\mathrm{N}$ which possesses the property $\operatorname{dim} \mathrm{X}_{n}=\operatorname{dim} \mathrm{Y}_{n}<\infty$.

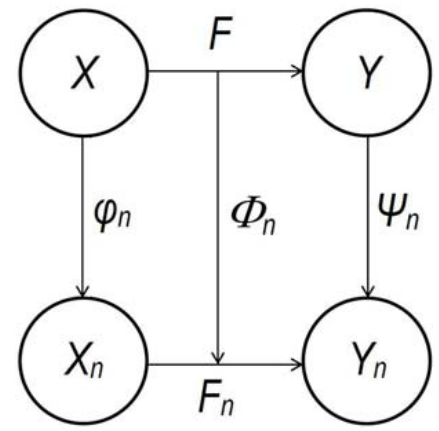

Fig. (1). The general scheme of numerical methods.

Assumption 6 Let us apply the discretization D to problem $\mathrm{P}$. We assume that $F_{n}$ is continuous on some ball $B_{R}\left(\varphi_{n}(\bar{u})\right)$.

Definition 7 The element $e_{n}=\varphi_{n}(\bar{u})-\bar{u}_{n} \in \mathrm{X}_{n}$ is called global discretization error.

Definition 8 The discretization $D$ applied to problem $P$ is called convergent if

$\lim \left\|e_{n}\right\|_{\mathrm{x}_{n}}=0$

holds.

Definition 9 The element $l_{n}(v)=F_{n}\left(\varphi_{n}(v)\right)-\psi_{n}(F(v)) \in Y_{n} \quad$ is called local discretization error at the element $v$. The local discretization error on the solution, i.e., $l_{n}(\bar{u})=F_{n}\left(\varphi_{n}(\bar{u})\right)-\psi_{n}(F(\bar{u}))=F_{n}\left(\varphi_{n}(\bar{u})\right)$, is called local discretization error.

Definition 10 The discretization $D$ applied to problem $\mathrm{P}$ is called consistent on the element $v \in D$ if

- $\varphi_{n}(v) \in \mathrm{D}_{n}$ holds from some index,

- the relation

$\lim \left\|l_{n}(v)\right\|_{Y_{n}}=0$

holds.

\section{T-STABILITY AND ITS APPLICATIONS}

In the 1980's V. A. Trenogin laid down the foundations of this topic. Namely, by giving the definition of T-stability, the EE method is considered on a uniform grid, and its Tstablility for the initial-value problem is proven. First we consider Trenogin's stability definition [8].

Definition 11 The discretization $D$ is called stable in Trenogin's sense (T-stable) if there exists a continuous, strictly monotonically increasing function $\omega(s)$, defined for $s \geq 0$, such that $\omega(0)=0$ and $\omega(\infty)=\infty$, and

$$
\begin{aligned}
& \omega\left(\left\|v_{n}^{1}-v_{n}^{2}\right\|_{\mathrm{X}_{n}}\right) \leq\left\|F_{n}\left(v_{n}^{1}\right)-F_{n}\left(v_{n}^{2}\right)\right\|_{\mathrm{Y}_{n}} \\
& \text { holds for all } v_{n}^{1}, v_{n}^{2} \in \mathrm{D}_{n} .
\end{aligned}
$$


Several theoretical results derived from Definition 11 (convergence of the numerical method, uniqueness of the discrete solution) can be found in [8].

In our consideration we suppose that $\varphi_{n} \in \operatorname{Lin}\left(\mathrm{X}, \mathrm{X}_{n}\right)$.

Definition 12 The sequence of $\|\cdot\|_{X}$ norms is called consistent to the norm $\|\cdot\|_{X}$, when for an arbitrary $v \in X$ the relation

$$
\lim \left\|\varphi_{n}(v)\right\|_{\mathrm{x}_{n}}=\|v\|_{\mathrm{X}}
$$

holds.

Remark 13 In most cases this condition is automatically satisfied.

For consistent norms the following property is valid.

Lemma 14 When the norms $\|\cdot\|_{\mathrm{X}_{n}}$ are consistent to the norm \|\|$_{\mathrm{X}}$, then the relation $v=0$ is valid if and only if $\lim \left\|\varphi_{n}(v)\right\|_{\mathrm{X}_{n}}=0$.

Proof. We consider two cases.

- If $v=0$, then $\lim \left\|\varphi_{n}(v)\right\|_{\mathrm{X}_{n}}=\|v\|_{\mathrm{X}}=0$;

- If $\lim \left\|\varphi_{n}(v)\right\|_{\mathrm{X}_{n}}=0$, then $\|v\|_{\mathrm{X}}=0$ and hence, $v=0$.

Remark 15 Generally, when in the spaces $\mathrm{X}_{n}$ $\lim \left\|\varphi_{n}(v)\right\|_{\mathrm{X}_{n}}=0$ if and only if $v=0$, we say that the spaces $\mathrm{X}_{n}$ are regularly normed. Hence, when $\|\cdot\|_{\mathrm{X}_{n}}$ is consistent to the norm $\|\cdot\|_{\mathrm{X}}$, then $\mathrm{X}_{n}$ are regularly normed spaces.

Theorem 16 Suppose that $\|\cdot\|_{x}^{\bullet}$

- the sequence of norms $\|\cdot\|_{X_{n}}$ is consistent to the norm

- there exists a solution to the problem (1)-(2),

- the discretization $\mathrm{D}$ is consistent and T-stable at the element $\bar{u}$.

Then

- $\bar{u}$ is unique,

- for any $n \in \mathrm{N}$ the discrete solution $\bar{u}_{n}$ is unique,

- the numerical method is convergent.

Proof.

- Let $v_{1}, v_{2}$ be solutions of (1) and assume that for these elements the relations

$$
\lim _{n \rightarrow \infty}\left\|F_{n}\left(\varphi_{n}\left(v_{1}\right)\right)\right\|_{Y_{n}}=0 ; \lim _{n \rightarrow \infty}\left\|F_{n}\left(\varphi_{n}\left(v_{2}\right)\right)\right\|_{Y_{n}}=0
$$

hold. Then

$$
\begin{aligned}
& \left\|\varphi_{n}\left(v_{1}-v_{2}\right)\right\|_{\mathrm{X}_{n}} \leq \omega^{-1}\left(\left\|F_{n}\left(\varphi_{n}\left(v_{1}\right)\right)-F_{n}\left(\varphi_{n}\left(v_{2}\right)\right)\right\|\right)_{\mathrm{Y}_{n}} \leq \\
& \leq \omega^{-1}\left(\left\|F_{n}\left(\varphi_{n}\left(v_{1}\right)\right)\right\|_{Y_{n}}+\left\|F_{n}\left(\varphi_{n}\left(v_{2}\right)\right)\right\|_{Y_{n}}\right) \rightarrow 0 \quad \text { if } \quad n \rightarrow \infty .
\end{aligned}
$$

Hence, we get

$$
\lim _{n \rightarrow \infty}\left\|\varphi_{n}\left(v_{1}-v_{2}\right)\right\|_{\mathrm{X}_{n}}=0 \text {. }
$$

The solution is unique, due to the fact that the finite dimensional spaces $\mathrm{X}_{n}$ are regularly normed.

- Let $v_{1}^{n}$ and $v_{2}^{n}$ be two solutions of (2). Substituting into (5), we get

$$
\begin{aligned}
& 0=\left\|F_{n}\left(v_{1}^{n}\right)-F_{n}\left(v_{2}^{n}\right)\right\|_{\mathrm{Y}_{n}} \geq \omega\left(\left\|v_{1}^{n}-v_{2}^{n}\right\|_{\mathrm{X}_{n}}\right) \geq 0, \\
& \text { i.e., } \quad \omega\left(\left\|v_{1}^{n}-v_{2}^{n}\right\|_{\mathrm{X}_{n}}\right)=0 \text {. From the norm property it }
\end{aligned}
$$
follows that $v_{1}^{n}=v_{2}^{n}$.

- Let $v_{1}$ and $v_{1}^{n}$ be solutions of (1) and (2), respectively. From the Definition 11 we gain

$$
\left\|v_{1}^{n}-\varphi\left(v_{1}\right)\right\|_{\mathrm{x}_{n}} \leq \omega^{-1}\left(\left\|F_{n}\left(v_{1}^{n}\right)-F_{n}\left(\varphi\left(v_{1}\right)\right)\right\|\right)_{Y_{n}}=\omega^{-1}\left(\left\|F_{n}\left(\varphi\left(v_{1}\right)\right)\right\|\right)_{Y_{n}},
$$

where we have used the consistency and also the continuity of the function $\omega^{-1}$ at the point $t=0$, i.e., it approaches zero when $n$ tends to $\infty$.

\subsection{How to Verify T-Stability for an Initial-Value Prob- lem?}

In this part we revise Definition 11 from the application point of view. Consider the well-known autonomous initialvalue problem:

$$
\begin{aligned}
& u^{\prime}(t)=f(u(t)) \\
& u(0)=u_{0},
\end{aligned}
$$

where $t \in[0,1], u_{0} \in \mathrm{R}$ and $f: \mathrm{R} \rightarrow \mathrm{R}$ is a Lipschitz continuous function. In the usual way a non-autonomous initial-value problem can be written in an autonomous form, where right-hand side function of the original problem is denoted by $f(t, u(t))$.

In the sense of Definition (1), (2) and (3) the operators $F, F_{n}, \varphi_{n}, \psi_{n}, \Phi_{n}$ and the spaces $\mathrm{X}, \mathrm{Y}, \mathrm{X}_{n}, \mathrm{Y}_{n}$ are defined as follows:

- $\mathrm{X}=C^{1}[0,1],\|u\|_{\mathrm{X}}=\max _{t \in[0,1]}|u(t)|$,

- $\mathrm{Y}=C[0,1] \times \mathrm{R},\left\|\left(\begin{array}{c}u \\ u_{0}\end{array}\right)\right\|_{\mathrm{Y}}=\max _{t \in 0,1]}(|u(t)|)+\left|u_{0}\right|$,

- $\quad F(u)=\left(\begin{array}{c}u^{\prime}(t)-f(u(t)) \\ u(0)-u_{0}\end{array}\right)$.

Applying the given one-step method to problem (7)-(8), for $n \in \mathrm{N}$ we define the following sequence of triplets:

- $\quad \mathrm{X}_{n}=\mathrm{R}^{n+1}, v_{n}=\left(v_{0}, v_{1}, \ldots, v_{n}\right) \in \mathrm{X}_{n}:\left\|v_{n}\right\|_{\mathrm{X}_{n}}=\max _{i=0, \ldots, n}\left|v_{i}\right|$,

- $\mathrm{Y}_{n}=\mathrm{R}^{n+1}, y_{n}=\left(y_{0}, y_{1}, \ldots, y_{n}\right) \in \mathrm{Y}_{n}:\left\|y_{n}\right\|_{Y_{n}}=\left|y_{0}\right|+\max _{i=1, \ldots, n}\left|y_{i}\right|$,

- The definition of the operator $F_{n}: \mathrm{R}^{n+1} \rightarrow \mathrm{R}^{n+1}$ depends on the chosen one-step method.

We define the pair of the restriction operators as follows: 
- For any $x \in \mathrm{X}$ we put $\left[\varphi_{n}(x)\right]_{i}=x\left(t_{i}\right), i=0,1, \ldots, n$,

- For any $y \in Y$ we put $\left[\psi_{n}(y)\right]_{i}= \begin{cases}y\left(t_{i-1}\right), & i=1, \ldots, n, \\ y\left(t_{0}\right), & i=0 .\end{cases}$

- The definition of the operator $\Phi_{n}(F)\left(\varphi_{n}\right): C^{1}[0,1] \rightarrow \mathrm{R}^{n+1}$ depends on the chosen one-step method.

Remark 17 In the following section we will define precisely the operators $F_{n}$ and $\Phi_{n}$ for the explicit and implicit one-step methods.

To verify the T-stability of a given method applied to problem (7)-(8), we consider the equation

$$
F_{n}\left(x_{n}+z_{n}\right)-F_{n}\left(x_{n}\right)=y_{n}
$$

where $x_{n}$ is some parameter, and $z_{n}$ is unknown. If we can give an estimation in the form

$$
\left\|z_{n}\right\|_{\mathrm{X}_{n}} \leq \zeta\left(\left\|y_{n}\right\|_{Y_{n}}\right)
$$

where the properties of $\zeta(s)$ correspond with the properties of $\omega(s)$, then by the choice $\omega(s):=\zeta^{-1}(s)$ we prove the T-stability.

Let $x_{n}=x_{1}^{n}$, while $x_{n}+z_{n}=x_{2}^{n}$ in (9). Then $F_{n}\left(x_{2}^{n}\right)-F_{n}\left(x_{1}^{n}\right)=y_{n}$ and $x_{2}^{n}-x_{1}^{n}=z_{n}$. Based on estimation (10), we get

$$
\left\|x_{2}^{n}-x_{1}^{n}\right\|_{\mathrm{X}_{n}} \leq \zeta\left(\left\|F_{n}\left(x_{2}^{n}\right)-F_{n}\left(x_{1}^{n}\right)\right\|_{\mathrm{Y}_{n}}\right) \text {. }
$$

Because the inverse of $\zeta$ exists and it is strictly monotonically increasing, we have

$$
\xi^{-1}\left(\left\|x_{2}^{n}-x_{1}^{n}\right\|_{\mathrm{X}_{n}}\right) \leq\left\|F_{n}\left(x_{2}^{n}\right)-F_{n}\left(x_{1}^{n}\right)\right\|_{\mathrm{Y}_{n}} .
$$

This matches the stability estimation in Definition 11.

To verify stability, we have to prove that the estimation

$$
\left\|z_{n}\right\|_{\mathrm{X}_{n}} \leq c\left\|y_{n}\right\|_{\mathrm{Y}_{n}}=c\left(\max _{1 \leq i \leq n}\left|y_{i}\right|+\left|u_{0}\right|\right)
$$

holds.

\section{T-STABILITY OF ONE-STEP METHODS FOR THE INITIAL-VALUE PROBLEM}

In this section we generalize Trenogin's result. Namely, we show that, under a natural assumption, any explicit or implicit one-step method is T-stable for the initial-value problem on a non-uniform mesh, too.

Consider the non-uniform grid:

$$
\mathrm{G}_{n}:=\left\{h_{i}=t_{i}-t_{i-1}, i=1, \ldots, n, 0=t_{0}<t_{1}<\ldots<t_{n}=1\right\} .
$$
as

The general form of the one-step method can be written

$$
y_{i}=y_{i-1}+h_{i} \Lambda\left(t_{i-1}, y_{i-1}, y_{i}, h_{i}\right)
$$

where $\Lambda: R^{4} \mapsto R$ defines the given numerical method $N$. (We note that the function $\Lambda$ is called the increment function and can be interpreted as an estimate of the slope of $\left.y_{i}.\right)$ We will say that the methods are explicit if $\Lambda=\Lambda\left(t_{i-1}, y_{i-1}, h_{i}\right)$. In the other case the methods are implicit.

\subsection{Explicit One-Step Methods}

In this part we consider the case where the numerical method is explicit. To this aim, we define the operators $F_{n}$ and $\Phi_{n}$ as follows:

- $F_{n}: \mathrm{R}^{n+1} \rightarrow \mathrm{R}^{n+1}$ and for any $v_{n}=\left(v_{0}, v_{1}, \ldots, v_{n}\right) \in \mathrm{R}^{n+1}$ it acts as

$$
\left(F_{n}\left(v_{n}\right)\right)_{i}= \begin{cases}\frac{1}{h_{i}}\left(v_{i}-v_{i-1}\right)-\Lambda\left(t_{i-1}, v_{i-1}, h_{i}\right), & i=1, \ldots, n, \\ v_{0}-u_{0}, & i=0 .\end{cases}
$$

- In order to give $\Phi_{n}$, we define the mapping $\Phi_{n}(F)\left(\varphi_{n}\right): C^{1}[0,1] \rightarrow \mathrm{R}^{n+1}$ in the following way:

$$
\left[\left(\Phi_{n} F\right)\left(\varphi_{n} u\right)\right]_{i}= \begin{cases}\frac{u\left(t_{i}\right)-u\left(t_{i-1}\right)}{h_{i}}-\Lambda\left(t_{i-1}, u\left(t_{i-1}\right), h_{i}\right), & i=1, \ldots, n, \\ u\left(t_{0}\right)-u_{0}, & i=0 .\end{cases}
$$

Next we verify the T-stability property of the operator of the explicit one-step methods, given in (16).

In the sequel we assume that $\Lambda$ is a Lipschitz continuous function with respect to its second variable, by the constant $L_{\Lambda}$. It means there exists a constant $L_{\Lambda} \geq 0$ such that for arbitrary $s_{1}, s_{2} \in \mathrm{R}$ the estimation

$$
\left|\Lambda\left(t_{i-1}, s_{1}, h_{i}\right)-\Lambda\left(t_{i-1}, s_{2}, h_{i}\right)\right| \leq L_{\Lambda}\left|s_{1}-s_{2}\right|
$$

holds for $t_{i-1} \in \mathrm{G}_{n}$ and $0<h_{i}$.

Remark 18 The Lipschitz assumption (17) is obviously necessary in proving the convergence. For the explicit Runge-Kutta methods this condition can be guarantied directly: when the Lipschitz assumption for the function $f$ in (7) is valid, then the increment function $\Lambda$ of the eligible explicit Runge-Kutta method satisfies the condition (17). (For non-autonomous problems the same Lipschitz condition is w.r.t. the second variable.)

Substituting $F_{n}$ into (9) and (15), we gain

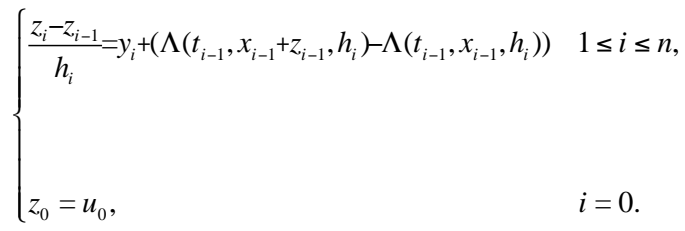

From (18), we get the estimation 


$$
\left|z_{i}\right| \leq\left(1+L_{\Lambda} h_{i}\right)\left|z_{i-1}\right|+h_{i}\left|y_{i}\right|, i=1, \ldots, n .
$$

\subsubsection{The case of $h_{i} \equiv h$}

For each index $i$ writing out (19) and applying it recursively, we get

$$
\begin{aligned}
& \left|z_{1}\right| \leq\left(1+L_{\Lambda} h\right)\left|u_{0}\right|+h\left\|y_{n}\right\|_{\infty} \\
& \left|z_{2}\right| \leq\left(1+L_{\Lambda} h\right)^{2}\left|u_{0}\right|+h\left\|y_{n}\right\|_{\infty}\left(1+\left(1+L_{\Lambda} h\right)\right) \\
& \vdots \\
& \left|z_{n}\right| \leq \underbrace{\left(1+L_{\Lambda} h\right)^{n}}_{I .}\left|u_{0}\right|+\left\|y_{n}\right\|_{\infty} \underbrace{h \sum_{k=0}^{n-1}\left(1+L_{\Lambda} h\right)^{k}}_{I I .} .
\end{aligned}
$$

In the next step we estimate the terms on the right-hand side of (20).

$$
\begin{aligned}
& I . \leq e^{L_{\Lambda} h n}=e^{L_{\Lambda}} \\
& I I . \leq h \sum_{k=0}^{n-1}\left(1+L_{\Lambda} h\right)^{k}=h \frac{\left(1+L_{\Lambda} h\right)^{n}-1}{1+L_{\Lambda} h-1} \leq h \frac{e^{L_{\Lambda} h n}-1}{L_{\Lambda} h}=\frac{e^{L_{\Lambda}}-1}{L_{\Lambda}}
\end{aligned}
$$

Then we get for the norm of $z_{n}$ the following estimation:

$$
\left\|z_{n}\right\|_{\mathrm{X}_{n}} \leq e^{L_{\Lambda}}\left|u_{0}\right|+\left\|y_{n}\right\|_{\infty} \frac{e^{L_{\Lambda}}-1}{L_{\Lambda}} \leq c\left\|y_{n}\right\|_{\mathrm{Y}_{n}}
$$

with the choice

$$
c=\max \left(e^{L_{\Lambda}}, \frac{e^{L_{\Lambda}}-1}{L_{\Lambda}}\right) .
$$

This implies the validity of the estimation (12).

Hence, we have proved the following statement.

Theorem 19 Under the condition (17), on a uniform grid the explicit one-step numerical method (14) is T-stable.

Therefore, on the basis of Remark 18, the following statement is true.

Corollary 20 For the Lipschitz continuous function $f$ on a uniform grid the explicit Runge-Kutta methods are Tstable.

Remark 21 For $\Lambda\left(t_{i-1}, x_{i-1}, h\right) \equiv f\left(t_{i-1}, x_{i-1}\right)$ we obtain the EE method on a uniform grid. Thus, Theorem 19 implies Trenogin's basic result. (The constant $c$ is the same which is given in [8].)

\subsubsection{The Case of a Non-Uniform Mesh}

When the grid is non-uniform, i.e., the step size is not constant, we can use the previous formula. Namely, for each index $i$ writing out (19) and applying it recursively, we get

$$
\left|z_{1}\right| \leq\left(1+L_{\Lambda} h_{1}\right)\left|u_{0}\right|+h_{1}\left\|y_{n}\right\|_{\infty}
$$

$$
\begin{gathered}
\left|z_{n}\right| \leq \underbrace{\left(1+L_{\Lambda} h_{n}\right) \cdot \ldots \cdot\left(1+L_{\Lambda} h_{1}\right)\left|u_{0}\right|}_{I \cdot}+ \\
+\left\|y_{n}\right\|_{\infty} \underbrace{\sum_{k=1}^{n} h_{k}\left(1+L_{\Lambda} h_{k+1}\right) \cdot \ldots \cdot\left(1+L_{\Lambda} h_{n}\right) .}_{\text {kn= }}
\end{gathered}
$$

In the next step we estimate the terms on the right-hand side of (21).

$$
\begin{aligned}
& I . \leq e^{L_{\Lambda} h_{n}} \cdot \ldots \cdot e^{L_{\Lambda} h_{1}}=e^{L_{\Lambda}\left(h_{n}+\ldots+h_{1}\right)}=e^{L_{\Lambda}} \\
& I I . \leq \sum_{k=1}^{n} h_{k} e^{\left(1-t_{k}\right) L_{\Lambda}}=e^{L_{\Lambda}} \sum_{k=1}^{n} h_{k}\left(e^{-L_{\Lambda}}\right)^{t_{k}}<e^{L_{\Lambda}} \sum_{k=1}^{n} \int_{k-1}^{t_{k}}\left(e^{-L_{\Lambda}}\right) d t= \\
& =e^{L_{\Lambda}} \int_{0}^{1}\left(e^{-L_{\Lambda}}\right) d t=e^{L_{\Lambda}}\left[-\frac{1}{L_{\Lambda}} e^{-L_{\Lambda} t^{t}}\right]_{0}^{1}=\frac{e^{L_{\Lambda}}-1}{L_{\Lambda}}
\end{aligned}
$$

Then for the norm of $z_{n}$ we get the following estimation:

$$
\left\|z_{n}\right\|_{\mathrm{X}_{n}} \leq e^{L_{\Lambda}}\left|u_{0}\right|+\left\|y_{n}\right\|_{\infty} \frac{e^{L_{\Lambda}}-1}{L_{\Lambda}} \leq c\left\|y_{n}\right\|_{\mathrm{Y}_{n}}
$$

with the choice

$$
c=\max \left(e^{L_{\Lambda}}, \frac{e^{L_{\Lambda}}-1}{L_{\Lambda}}\right) .
$$

Therefore, we obtain the estimation in the form (12).

Hence, like for uniform meshes, we have proved the following statements.

Theorem 22 Under the condition (17), on a non-uniform grid the explicit one-step numerical method (14) is T-stable.

Corollary 23 For a Lipschitz continuous function $f$ on a non-uniform grid the explicit Runge-Kutta methods are Tstable.

\subsection{Implicit One-Steps Methods}

In this part we move on to the consideration of implicit one-step methods, particularly, to the investigation of implicit Runge-Kutta methods.

As we have already mentioned in Remark 17, we have to define the operators $F_{n}$ and $\Phi_{n}$. In the sequel the operators are given as follows:

- $F_{n}: \mathrm{R}^{n+1} \rightarrow \mathrm{R}^{n+1}$ and for any $v_{n}=\left(v_{0}, v_{1}, \ldots, v_{n}\right) \in \mathrm{R}^{n+1}$ it acts as

$$
\left(F_{n}\left(v_{n}\right)\right)_{i}= \begin{cases}\frac{1}{h_{i}}\left(v_{i}-v_{i-1}\right)-\Lambda\left(t_{i-1}, v_{i-1}, v_{i}, h_{i}\right), & i=1, \ldots, n, \\ v_{0}-u_{0}, & i=0 .\end{cases}
$$

- In order to give $\Phi_{n}$, we define the mapping $\Phi_{n}(F)\left(\varphi_{n}\right): C^{1}[0,1] \rightarrow \mathrm{R}^{n+1}$ in the following way:

$$
\left[\left(\Phi_{n} F\right)\left(\varphi_{n} u\right)\right]_{i}= \begin{cases}\frac{u\left(t_{i}\right)-u\left(t_{i-1}\right)}{h_{i}}-\Lambda\left(t_{i-1}, u\left(t_{i-1}\right), u\left(t_{i}\right), h_{i}\right), & 1 \leq i \leq n, \\ u\left(t_{0}\right)-u_{0}, & i=0 .\end{cases}
$$


Hence, we have to verify the T-stability of the operator, given in (23). In the following we suppose that $\Lambda$ is a Lipschitz continuous function with respect to its second and third variable, by the constants $L_{\Lambda_{1}}$ and $L_{\Lambda_{2}}$. It means there exist $L_{\Lambda_{1}}, L_{\Lambda_{2}} \geq 0$ constants, such that for arbitrary $s_{1}, s_{2}, p_{1}, p_{2} \in \mathrm{R}$ the estimation

$$
\left|\Lambda\left(t_{i-1}, s_{1}, p_{1}, h_{i}\right)-\Lambda\left(t_{i-1}, s_{2}, p_{2}, h_{i}\right)\right| \leq L_{\Lambda_{1}}\left|s_{1}-s_{2}\right|+L_{\Lambda_{2}}\left|p_{1}-p_{2}\right|
$$

holds for $t_{i-1} \in \mathrm{G}_{n}$ and $0<h_{i}$.

Remark 24 For the implicit Runge-Kutta methods the Lipschitz assumption (24) can be also guaranteed directly: when the Lipschitz assumption for the function $f$ in (7) is valid, then the increment function $\Lambda$ of the eligible implicit Runge-Kutta method, for a sufficiently small $h$, satisfies the condition (24).

Substituting $F_{n}$ into (9), (22) and using the Lipschitz condition (24), we get the estimation

$$
\left|z_{i}\right| \leqslant\left|z_{i-1}\right|+h_{i}\left|y_{i}\right|+h_{i}\left(L_{\Lambda_{1}}\left|z_{i-1}\right|+L_{\Lambda_{2}}\left|z_{i}\right|\right), i=1, \ldots, n .
$$

Hence, we get

$$
\left|z_{i}\right| \leq \frac{1+h_{i} L_{\Lambda_{1}}}{1-h_{i} L_{\Lambda_{2}}}\left|z_{i-1}\right|+\frac{1}{1-h_{i} L_{\Lambda_{2}}} h_{i}\left|y_{i}\right|, i=1, \ldots, n .
$$

We give an estimation for $\frac{1}{1-h_{i} L_{\Lambda_{2}}}$. If $h_{i} L_{\Lambda_{2}} \in[0,0.5]$ for all $i$, then we can write this expression as

$$
1 \leq \frac{1}{1-h_{i} L_{\Lambda_{2}}}=1+h_{i} L_{\Lambda_{2}}+\left(h_{i} L_{\Lambda_{2}}\right)^{2} \frac{1}{1-h_{i} L_{\Lambda_{2}}} .
$$

Obviously, for the values $\left.h_{i} L_{\Lambda_{2}} \in 0,0.5\right]$ the following estimation holds:

$$
\frac{\left(h_{i} L_{\Lambda_{2}}\right)^{2}}{1-h_{i} L_{\Lambda_{2}}} \leq h_{i} L_{\Lambda_{2}} .
$$

Therefore, we have the upper bound

$$
\frac{1}{1-h_{i} L_{\Lambda_{2}}} \leq 1+2 h_{i} L_{\Lambda_{2}} \leq \exp \left(2 h_{i} L_{\Lambda_{2}}\right)
$$

Thus, we can write equation (25) in the form:

$$
\left|z_{i}\right| \xi\left(1+h_{i} L_{\Lambda_{1}}\right)\left(1+2 h_{i} L_{\Lambda_{2}}\right)\left|z_{i-1}\right|+\left(1+2 h_{i} L_{\Lambda_{2}}\right) h_{i}\left|y_{i}\right|, i=1, \ldots, n .
$$

\subsubsection{The Case of $h_{i} \equiv h$}

For each index $i$ writing out (26) and applying it recursively, we get

$$
\begin{aligned}
& \left|z_{1} \leqslant\left(1+h L_{\Lambda_{1}}\right)\left(1+2 h L_{\Lambda_{2}}\right)\right| u_{0}\left|+\left(1+2 h L_{\Lambda_{2}}\right) h\right| y_{n} \|_{\infty} \\
& \vdots \\
& \left|z_{n}\right| \leqslant \underbrace{\left(\left(1+h L_{\Lambda_{1}}\right)\left(1+2 h L_{\Lambda_{2}}\right)\right)^{n}+\left\|y_{n}\right\|_{\infty} \underbrace{\sum_{k=1}^{n}\left(1+h L_{\Lambda_{1}}\right)^{k-1}\left(1+h L_{\Lambda_{2}}\right)^{k}}_{k=1} .}_{l .}
\end{aligned}
$$

In the next step we estimate the terms on the right-hand side of (27).

$$
\begin{aligned}
& I . \leq e^{h n L_{\Lambda_{1}}} e^{2 h n L_{\Lambda_{2}}}=e^{L_{\Lambda_{1}}} e^{2 L_{\Lambda_{2}}}=e^{L_{\Lambda_{1}}+2 L_{\Lambda_{2}}} \\
& I I . \leq h \sum_{k=1}^{n}\left[\left(1+h L_{\Lambda_{1}}\right)\left(1+h L_{\Lambda_{2}}\right)\right]^{k} \leq h \frac{\left[\left(1+h L_{\Lambda_{1}}\right)\left(1+h L_{\Lambda_{2}}\right)\right]^{n+1}-1}{\left(1+h L_{\Lambda_{1}}\right)\left(1+h L_{\Lambda_{2}}\right)-1} \leq \\
& \leq h \frac{e^{L_{\Lambda_{1}}+2 L_{\Lambda_{2}}}-1}{h L_{\Lambda_{1}}+2 h^{2} L_{\Lambda_{1}} L_{\Lambda_{2}}+2 h L_{\Lambda_{2}}} \leq \frac{e^{L_{\Lambda_{1}}+2 L_{\Lambda_{2}}}-1}{L_{\Lambda_{1}}+2 L_{\Lambda_{2}}}
\end{aligned}
$$

Then for the norm of $z_{n}$ we get the following estimation:

$$
\left\|z_{n}\right\|_{\mathrm{X}_{n}} \leq e^{L_{\Lambda_{1}}+2 L_{\Lambda_{2}}}\left|u_{0}\right|+\left\|y_{n}\right\|_{\infty} \frac{e^{L_{\Lambda_{1}}+2 L_{\Lambda_{2}}}-1}{L_{\Lambda_{1}}+2 L_{\Lambda_{2}}} \leq c\left\|y_{n}\right\|_{Y_{n}}
$$

with the choice

$$
c=\max \left(e^{L_{\Lambda_{1}}+2 L_{\Lambda_{2}}}, \frac{e^{L_{\Lambda_{1}}+2 L_{\Lambda_{2}}}-1}{L_{\Lambda_{1}}+2 L_{\Lambda_{2}}}\right) .
$$

Hence, we obtain the estimation (12), which shows the validity of the following statements.

Theorem 25 Under the condition (24) on a uniform grid, the implicit one-step numerical method (23) is T-stable.

Corollary 26 For a Lipschitz continuous function $f$ on a uniform grid the implicit Runge-Kutta methods are Tstable.

\subsubsection{The Case of Non-Uniform Meshes}

In the sequel we consider the implicit one-step methods on non-uniform meshes. Similarly to the previous case, for each index $i$ writing out (26) and applying it recursively, we get

$$
\begin{aligned}
& \left|z_{1}\right| \leqslant\left(1+h_{1} L_{\Lambda_{1}}\right)\left(1+2 h_{1} L_{\Lambda_{2}}\right)\left|u_{0}\right|+\left(1+2 h_{1} L_{\Lambda_{2}}\right) h_{1}|| y_{n} \|_{\infty} \\
& \left|z_{n}\right| \leq \underbrace{\left(1+h_{n} L_{\Lambda_{1}}\right) \ldots .\left(1+h_{1} L_{\Lambda_{1}}\right)\left(1+2 h_{n} L_{\Lambda_{2}}\right) \ldots .\left(1+2 h_{1} L_{\Lambda_{2}}\right)}_{\text {. }}+
\end{aligned}
$$

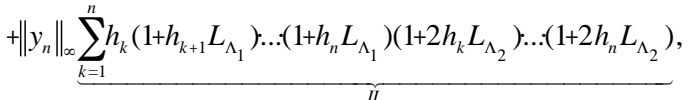

In the next step we estimate the terms on the right-hand side of (28).

$$
\begin{aligned}
& I . \leq e^{L_{\Lambda_{1}}\left(h_{n}+\ldots+h_{1}\right)} e^{2 L_{\Lambda_{2}}\left(h_{n}+\ldots+h_{1}\right)}=e^{L_{\Lambda_{1}}+2 L_{\Lambda_{2}}} \\
& I I . \leq \sum_{k=1}^{n} h_{k} e^{\left(1-t_{k}\right) L_{\Lambda_{1}}} e^{\left(1-t_{k-1} 12 L_{\Lambda_{2}}\right.}=e^{L_{\Lambda_{1}}+2 L_{\Lambda_{2}}} \sum_{k=1}^{n} h_{k}\left(e^{-L_{\Lambda_{1}}}\right)^{t_{k}}\left(e^{-2 L_{\Lambda_{2}}}\right)^{t_{k-1}}< \\
& <e^{L_{\Lambda_{1}}+2 L_{\Lambda_{2}}} \sum_{k=1}^{n} \int_{t_{k-1}}^{t_{k}}\left(e^{-\left[L_{\Lambda_{1}}+2 L_{\Lambda_{2}}\right]}\right)^{t} d t=e^{L_{\Lambda_{1}}+2 L_{\Lambda_{2}}} \int_{0}^{1}\left(e^{-\left[L_{\Lambda_{1}}+2 L_{\Lambda_{2}}\right]}\right)^{t} d t= \\
& =\frac{e^{L_{\Lambda_{1}}+2 L_{\Lambda_{2}}}-1}{L_{\Lambda_{1}}+2 L_{\Lambda_{2}}}
\end{aligned}
$$

Then for the norm of $z_{n}$ we get the estimation 


$$
\left\|z_{n}\right\|_{\mathrm{X}_{n}} \leq e^{L_{\Lambda_{1}}+2 L_{\Lambda_{2}}}\left|u_{0}\right|+\left\|y_{n}\right\|_{\infty} \frac{e^{L_{\Lambda_{1}}+2 L_{\Lambda_{2}}}-1}{L_{\Lambda_{1}}+2 L_{\Lambda_{2}}} \leq c\left\|y_{n}\right\|_{Y_{n}}
$$

with the choice

$$
c=\max \left(e^{L_{\Lambda_{1}}+2 L_{\Lambda_{2}}}, \frac{e^{L_{\Lambda_{1}}+2 L_{\Lambda_{2}}}-1}{L_{\Lambda_{1}}+2 L_{\Lambda_{2}}}\right) .
$$

Hence, we can write an estimation in the form (12) again. Hence, we can formulate our main result in the form of the following statements.

Theorem 27 Under the condition (24) on a non-uniform grid, the implicit one-step numerical method (23) is T-stable.

Corollary 28 For a Lipschitz continuous function $f$ on a non-uniform grid, the implicit Runge-Kutta methods are Tstable.

\section{SUMMARY}

In this paper we have considered initial-value problems in an abstract (Banach space) setting. As we have seen in [8] and also in our previous work [3], the explicit Euler method on a uniform grid is S- and T-stable for the initial-value problem with a Lipschitz continuous function $f$. In the present work we have shown that under the same condition for the function $f$, the T-stability is preserved for more general Runge-Kutta methods, namely, for any explicit and implicit methods. We have also shown that the stability results are valid for both uniform and non-uniform meshes, too. We have also shown that under some natural condition for the numerical method $\mathrm{N}$, which is automatically satisfied for Runge-Kutta methods, the results remain valid for arbitrary one-step methods.

As we can see in (Table 1), we get that the same stability constant $c$ can be achieved for the different meshes, i.e., from this aspect there is no distinction between the cases of uniform and non-uniform grids.

The obtained T-stability together with the consistency ensures the convergence. The consistency of the one-step methods can be given by the following two properties (see, e.g., [5]): the Lipschitz condition and the increment function $\Lambda$ for the function $f=0$ should be identically zero, i.e., $\Lambda\left(t_{i-1}, v_{i-1}, v_{i}, h_{i}\right)=0$.

Recently we have introduced the local version of $\mathrm{T}$ stability (called locT-stability) and we have proved a similar theorem to Theorem 16. This motivates that in the upcoming works it is worth dealing with the case where the right-hand side function $f$ is a locally Lipschitz function. The other direction of the future work is to extend our results to multistep methods.

Table 1. T-Stability Constants of the Different Cases

\begin{tabular}{|c|c|c|}
\hline & Explicit one-step method & Implicit one-step method \\
\hline \hline$h$ & $\max \left(e^{L_{\Lambda}}, \frac{e^{L_{\Lambda}}-1}{L_{\Lambda}}\right)$ & $\max \left(e^{L_{\Lambda_{1}}+2 L_{\Lambda_{2}}}, \frac{e^{L_{\Lambda_{1}}+2 L_{\Lambda_{2}}}-1}{L_{\Lambda_{1}}+2 L_{\Lambda_{2}}}\right)$ \\
\hline$h_{i}$ & $\max \left(e^{L_{\Lambda}}, \frac{e^{L_{\Lambda}}-1}{L_{\Lambda}}\right)$ & $\max \left(e^{L_{\Lambda_{1}}+2 L_{\Lambda_{2}}}, \frac{e^{L_{\Lambda_{1}}+2 L_{\Lambda_{2}}}-1}{L_{\Lambda_{1}}+2 L_{\Lambda_{2}}}\right)$ \\
\hline
\end{tabular}

\section{CONFLICT OF INTEREST}

The author(s) confirm that this article content has no conflicts of interest.

\section{ACKNOWLEDGEMENT}

The work of the first author was supported by the Hungarian Research Grant OTKA K67819.

\section{REFERENCES}

[1] Charney J G, Fjortoft R, von Neumann J. Numerical Integration of the barotropic vorticity equation. Tellus 1950; 2: 237-54.

[2] Courant R, Friedrichs K, Lewy $H$. Über die partiellen Differenzengleichungen der mathematischen Physik. Mathematische Annalen 1928; 100: 32-74.

[3] Faragó I, Mincsovics ME, Fekete I. Notes on the basic notions in nonlinear numerical analysis, In: Proc. 9'th Coll. Qualitative Theory of Diff. Equ., Szeged, June 28-July 1, 2011, E. J. Qualitative Theory of Diff. Equ., No. 6, pp. 2012, 1-22.

[4] Keller H B. Approximation Methods for Nonlinear Problems with Application to Two-Point Boundary Value Problems. Math Comput 1975; 130: 464-74.

[5] Lambert J D. Numerical methods for ordinary differential systems, 1st ed, J Wiley and Sons, Chicetster, 1991.

[6] Lax P D, Richtmyer R D. Survey of the stability of linear finite difference equations, Comm. Pure Appl Math 1956; 9: 267-93.

[7] Stetter H J. Analysis of Discretization Methods for Ordinary Differential Equations, 1st ed., Springer, Berlin, 1973.

[8] Trenogin V A. Functional Analysis (in Russian), 1st ed., Nauka, Moscow, 1980. 